\title{
Optical anisotropy of InAs submonolayer quantum wells in a (311) GaAs matrix
}

\author{
Y. H. Chen* and Z. Yang ${ }^{\dagger}$ \\ Department of Physics, Hong Kong University of Science and Technology, Clearwater Bay, Kowloon, Hong Kong \\ Z. G. Wang, B. Xu, J. B. Liang, and J. J. Qian \\ Laboratory of Semiconductor Materials Science, Institute of Semiconductors, Chinese Academy of Sciences, \\ Box 912, Beijing 100083, China \\ (Received 11 April 1997)
}

\begin{abstract}
The in-plane optical anisotropy which comes from the heavy-hole and the light-hole transitions in InAs submonolayers inserted in a (311)-oriented GaAs matrix is studied by reflectance-difference spectroscopy (RDS). The steplike density of states obtained from RDS demonstrates that the ultrathin InAs layers should be regarded as two-dimensional quantum wells rather than isolated clusters, even for the sample with only $\frac{1}{3}$-ML InAs. The degree of anisotropy is found to be independent of the layer coverage, and is within the intrinsic anisotropy of (311)-oriented ultrathin quantum wells, indicating that there is little structural or strain anisotropy in the InAs submonolayer grown on a (311) GaAs surface. [S0163-1829(97)08835-8]
\end{abstract}

The optical properties of monolayer (ML) and submonolayer InAs imbedded in a GaAs matrix have recently attracted much attention. ${ }^{1-6}$ High photoluminescence (PL) efficiency, ${ }^{2}$ strong exciton oscillator strength, ${ }^{3}$ and huge binding energy ${ }^{4}$ were observed for submonolayer InAs structures and were attributed to the lateral quantum confinement due to the formation of quantum dots (QD's) or quantum wires. However, optical properties revealed by transmission spectra and time-resolved PL spectra suggested that the submonolayers behaved like two-dimensional quantum wells (QW's), ${ }^{5,6}$ even with only a $\frac{1}{2}$ ML of InAs. In this paper, we present the reflectance difference spectrum (RDS) and PL study of $1-, \frac{2}{3}-$, and $\frac{1}{3}$-ML InAs inserted in a (311) oriented GaAs matrix. From RDS we are able to extract an absorption anisotropy spectrum of the InAs layers. The steplike line shape of the spectra directly demonstrate that the ultrathin InAs layers should be regarded as QW rather than isolated QD's. A definite Stokes shift between the absorption and the PL peak further confirms the QW nature of the InAs layers. Furthermore, while the amplitude of the absorption anisotropy, $\Delta p$, is found to be linearly reduced with less InAs coverage, the degree of anisotropy, $\Delta p / p$, of the InAs layers is found to be independent of the coverage and within the intrinsic anisotropy of (311) oriented QW's. This indicates that unlike the InAs layers in (100) GaAs matrix, ${ }^{3}$ there is little structural and/or strain anisotropy in the InAs layers in (311) GaAs matrix.

RDS measures the difference of the complex reflection coefficient between two perpendicular directions in the surface plain and is usually employed for measuring the optical anisotropy of semiconductor surfaces/interfaces and linear electro-optical effects. ${ }^{7,8}$ Here we demonstrate that RDS can also be useful for the study of InAs submonolayers inserted in the GaAs matrix. Our RDS system is similar to that in Ref. 9 except that a Fourier transform spectrometer (FTS) was in place for the monochromator. The use of the FTS operating in the double-modulation slow-scan mode greatly improved the signal-to-noise ratio as compared to using a monochromator.
The sample was grown by conventional molecular-beam epitaxy (MBE) on (311)-oriented GaAs substrates. After the growth of a $0.5-\mu \mathrm{m} \mathrm{GaAs}$ buffer layer at $600{ }^{\circ} \mathrm{C}$ the substrate was cooled down to $450{ }^{\circ} \mathrm{C}$ and the ultrathin InAs layer was then deposited. The growth rate of GaAs was 0.7 $\mu \mathrm{m} / \mathrm{h}$ and that of InAs was $0.2 \mu \mathrm{m} / \mathrm{h}$. A growth interruption of 2 min were introduced before and after the InAs deposition. Finally, an $800-\AA \mathrm{GaAs}$ cap layer was grown at $600^{\circ} \mathrm{C}$. The samples were then placed in a variable-temperature cryostat for the RDS measurement from 300 to $80 \mathrm{~K}$.

Figure 1 shows the complex RD spectra $\Delta \rho$ $\equiv 2\left(r_{1}-r_{2}\right) /\left(r_{1}+r_{2}\right)$ for the $1-, \frac{2}{3}-$, and $\frac{1}{3}$-ML samples measured at room temperature (RT). Here $r_{1}$ and $r_{2}$ represent the reflectance of light polarized along the two in-plane principal axes, the [011] and the [233] directions, respectively. The solid curves represent the real RD spectra, while the dashed curves represent the imaginary RD spectra. The resonance of all three samples have the same line shape, but their amplitude decreases with decreasing InAs coverage. Figure 2

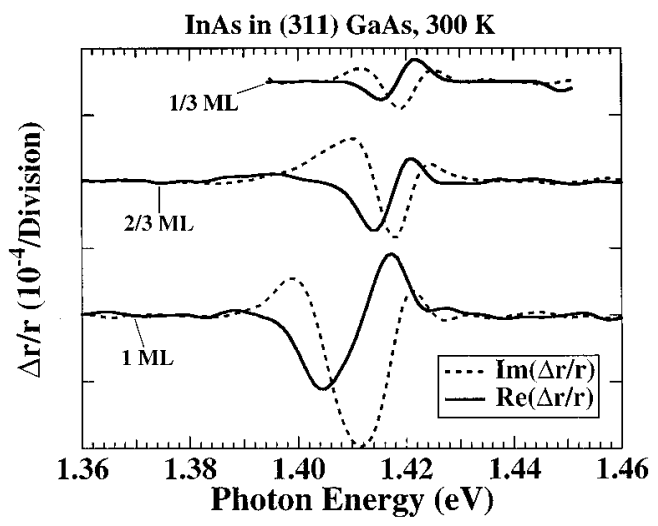

FIG. 1. The reflectance-difference spectra (RDS) of $1-, \frac{2}{3}-$, and $\frac{1}{3}$-ML InAs inserted in the (311) GaAs matrix measured at room temperature. The solid curves represent the real RD spectra while the dashed curves represent the imaginary spectra. Spectra are shifted vertically for clarity. 


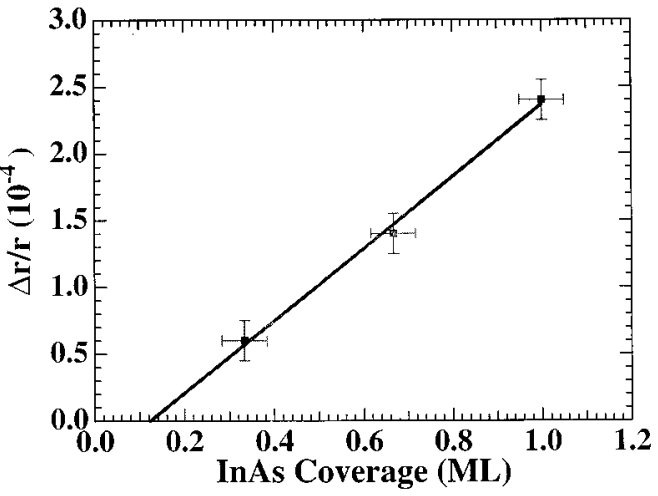

FIG. 2. The peak-to-peak amplitude of the resonance as a function of InAs coverage.

shows the well-defined linear dependence of the resonance amplitude on InAs coverage. An offset of $0.12 \mathrm{ML}$ is seen for a zero amplitude, indicating that the InAs coverage was probably overestimated by the same amount. This is possible since the InAs dosage was based on the growth rate on (100) GaAs. The resonance energy is blueshifted with reduced coverage due to enhanced confinement as frequently observed by PL. ${ }^{1,2}$ This clearly shows that the resonances indeed come from the InAs layer.

Figure 3 shows the complex RD spectra for the 1-ML sample measured at $80 \mathrm{~K}, 170 \mathrm{~K}$, and RT. In the plot the RT spectra have been shifted towards higher photon energy by $80 \mathrm{meV}$, and the $170 \mathrm{~K}$ ones have been shifted by $25 \mathrm{meV}$, to compensate for the temperature-dependent shift of the resonance energies. The spectra have also been shifted vertically for clarity. The dome-shaped resonance of the imaginary RD spectra gradually becomes steplike, and the two edges of the step become steeper at lower temperatures. The real RD spectra show corresponding resonances labeled (HH) (heavy hole) and (light hole) (LH) at the center of the step edges. This is expected because the real RD spectra are always the derivative, apart from a factor, of the corresponding imaginary RD spectra. ${ }^{10}$ The intensity of the " $\mathrm{HH}$ " and "LH" resonances increase slowly as the sample is cooled, and the intensities at $80 \mathrm{~K}$ are about two times of that at RT. A third sharp resonance, labeled "Ex," appears at higher

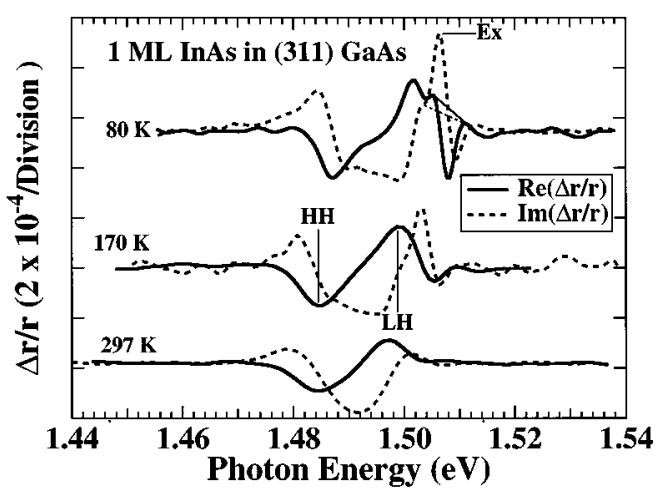

FIG. 3. Absorption anisotropy spectrum (solid curve) calculated from the RDS data in Fig. 3, and photoluminescence (dashed curve) of 1-ML sample at $80 \mathrm{~K}$. The "Ex" feature in the RDS has been removed for clarity.

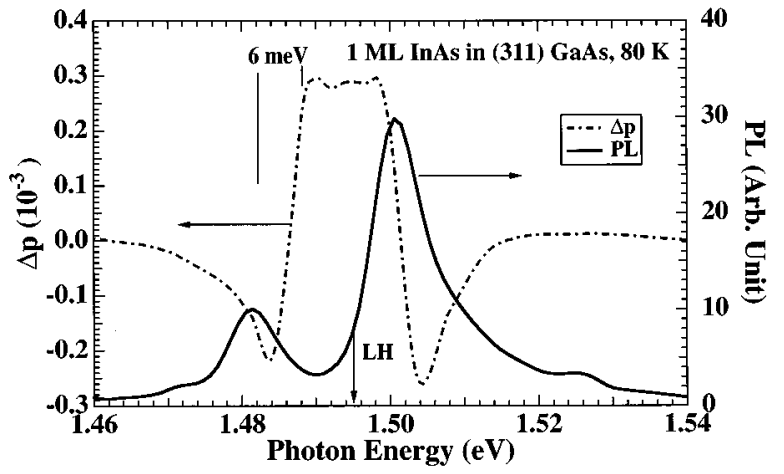

FIG. 4. The reflectance-difference spectra of 1-ML InAs sample measured at room temperature (RT), 170 and $80 \mathrm{~K}$. The solid curves represent the real part of RDS while the dashed curves represent the imaginary spectra. The RT spectra have been shifted to the right by $80 \mathrm{meV}$, while that of $170 \mathrm{~K}$ have been shifted by 25 $\mathrm{meV}$. The two thin lines for the $80-\mathrm{K}$ spectra show the portion of the spectra when the "Ex"' structure is removed.

energy at temperatures below $170 \mathrm{~K}$, and its intensity increases quickly with decreasing temperature. At $80 \mathrm{~K}$ the energy position of the three resonances is at 1.487, 1.502, and $1.507 \mathrm{eV}$, respectively. Similar "Ex" (exciton) resonance has been observed even in some of the (100)-oriented pure GaAs samples. Its energy position coincides with the GaAs bulk exciton at all temperatures, and its line shape is always very sharp. The origin of the resonance is not clear at present, but we speculate that the resonance comes from bulk excitons bound to anisotropic centers such as steps at the sample surface.

We now proceed to the analysis of the steplike feature in the RD spectra. Consider the InAs submonolayer as an optically anisotropic thin layer inserted in an isotropic medium, since GaAs shows no resonance structure in the energy range of interest, the RDS data are then related to the dielectric function anisotropy of the InAs layer by

$$
\Delta \rho=-\frac{4 \pi d i e^{i \phi} \Delta \varepsilon}{\lambda\left(\varepsilon_{s}-1\right)}
$$

with the phase shift $\phi$ due to the cap layer given by

$$
\phi=4 \pi n_{s} w / \lambda .
$$

Here $n_{s}\left(\varepsilon_{s}\right)$ is the refractive index (dielectric function) of GaAs, $\Delta \varepsilon$ is the difference of the complex dielectric function of the InAs layer the [011] and the [233] directions, $w$ is the thickness of the cap layer, $d$ is the effective thickness of the anisotropic layer, and $\lambda$ is the wavelength of light.

Defining the absorption probability of the InAs layer as $p=d \alpha$, in which $\alpha$ is the absorption coefficient of the InAs layer, we can then calculate $\Delta p(=d \Delta \alpha)$ directly from our RDS data according to Eq. (1). The results for the 1-ML sample at $80 \mathrm{~K}$ are shown in Fig. 4 as the solid curve. The realistic GaAs optical constants and $w=800 \AA$ were used in the calculations. Note that the "Ex" peak has been removed for clarity. The squarelike $\Delta p$ is the combined contribution of two steplike features, a positive one starting from 1.487 $\mathrm{eV}$ due to the $\mathrm{HH}$ subband to the conduction-subband transition, and a negative one starting from $1.502 \mathrm{eV}$ due to the 
LH subband to the conduction-subband transition. This is because in the (311) orientation the electronic transition anisotropy of the $\mathrm{HH}$ subbands and that of the $\mathrm{LH}$ subbands are opposite, ${ }^{11-13}$ as squarelike anisotropic absorption spectrum has been predicted theoretically for ( $n 11)$-oriented GaAs/ AlAs QW. ${ }^{12,13}$ The steplike features observed here are a clear manifestation of the two-dimensional density of states (DOS) of the InAs layer.

Having established that the DOS of the 1-ML InAs layer is two dimensional, we now proceed to the analysis of the $\frac{1}{3}$ and 2/3-ML InAs layers. Unfortunately, the position of the " $\mathrm{HH}$ " and "LH" resonances of these two layers are so close to that of the "Ex" resonance that it is impossible to separate them from the "Ex" resonance at low temperatures. However, the similar line shape of $\Delta \rho$ for all three samples at RT (see Fig. 1), where the contribution of GaAs exciton is negligible, shows that the three samples have the same 2D DOS. In fact, if the DOS is zero or one dimensional, we shall observe two peaks with opposite signs for the $\mathrm{HH}$ and the LH transitions in the imaginary part of $\Delta \rho$, not the single peak as we have seen in Fig. 1.

In order to compare our RDS data with other experimental and theoretical results, it is necessary to know the absorption possibility $p$ of the InAs layer. Two well-defined resonances, corresponding to that in the RD spectra, were also observed in the reflectance spectra at $80 \mathrm{~K}$ for the 1-ML sample. If the reflectivity of the discussed sample with (without) the InAs layer is $R\left(R_{0}\right)$, then $d\left(\varepsilon-\varepsilon_{s}\right)$ is related to $\left(R-R_{0}\right) / R_{0}$ by the same Eq. (1), except that $\Delta \rho$ should be replaced by $\left(R-R_{0}\right) / R_{0}$ and $\Delta \varepsilon$ replaced by $\left(\varepsilon-\varepsilon_{s}\right)$ while only the real part of the right side of the equation is retained. Our reflection data gives $\left(R-R_{0}\right) / R_{0}=0.008$ for both the $\mathrm{HH}$ and the LH transitions, from which we can estimate $p$ $=0.013$ in the 1-ML sample. This is in good agreement with the reported result, where $p=0.12$ was obtained by transmission for a sample with ten single ML of InAs separately inserted in (100) GaAs. ${ }^{6}$ From Fig. 4 and Eq. (1) we obtain $\Delta p=5 \times 10^{-4}$, and therefore $\Delta p / p=4 \%$ for both transitions, which agrees with the PL result (4\% for $\mathrm{HH}$ and $5 \%$ for LH) of a $100-\AA$ (311)-oriented GaAs/ $\mathrm{Al}_{x} \mathrm{Ga}_{1-x} \mathrm{As} \mathrm{QW},{ }^{11}$ and the calculated results (about 5\%) for the lowest HH subband in 200- $\AA$ (311)-oriented GaAs/AlAs QW. ${ }^{12}$ Theoretical study has shown that the optical anisotropy of non-(100)oriented QW's is determined by the intrinsic anisotropy of the Bloch wave functions of holes. In both cases of wide GaAs/ $\mathrm{Al}_{x} \mathrm{Ga}_{1-x} \mathrm{As}$ QW's and narrow InAs/GaAs QW's the hole wave functions consist of the Bloch wave functions of $\mathrm{GaAs}$ at the top of the valence bands. That is why the two cases show similar results as we have observed.

Figure 4 also shows the PL spectrum (dashed curve) measured at $80 \mathrm{~K}$. The peak at $1.481 \mathrm{eV}$ is due to the $\mathrm{HH}$ transition, and the peak at $1.501 \mathrm{eV}$ is assigned to the bound exciton of bulk GaAs according to the excitation power dependence of its intensity. The arrow at $1.495 \mathrm{eV}$ indicates the position of the LH transition PL peak at $80 \mathrm{~K}$ determined from the PL peak which could only be observed at $10 \mathrm{~K}$. The Stokes shift of about $6 \mathrm{meV}$ is obtained for both the $\mathrm{HH}$ and the LH transitions. Since the Stokes shift is a characteristic of quantum well, our PL results again confirm the 2D nature of the submonolayer InAs samples.
The dependence of the anisotropy on the InAs coverage can also be explained by the QW model. It is clearly seen from Fig. 2 that $\Delta \rho$, and therefore $\Delta p$, has a linear dependence on the InAs coverage $\Theta$, and therefore the effective thickness $d$, since $d$ should be proportional to $\Theta$. The value of $\Delta \alpha$ is then about the same for all three samples. Since the absorption coefficient $\alpha$ of the InAs layer is expected to be the same for all three samples, $\Delta \alpha / \alpha$ and therefore $\Delta p / p$, or the degree of anisotropy, is about the same for all three samples. Using the standard eight-band $\mathbf{k} \cdot \mathbf{p}$ formalism we have calculated the anisotropic transition probability between the electron and the hole subbands in (311) GaAs/ InAs QW's, and found that below $10 \AA$ the optical anisotropy is independent of the well width. This result is not in contradiction with the existing theoretical results, ${ }^{12,13}$ in which the in-plane optical anisotropy of (311) GaAs/AlAs QW's increases with the decrease of well width. This is because the magnitude of the anisotropy depends on the energy difference between the hole subbands and the $\Gamma_{7}$ bands of the constituent materials, and in the relatively wide QW's this energy difference depends on the well width. In the ultrathin InAs QW cases the hole subbands are always very near the GaAs $\Gamma_{8}$ valence-band edge and far away from the $\Gamma_{7}$ band. The effects of the split-off valence band is therefore almost fixed and the degree of anisotropy remains unchanged when the InAs coverage changes from 1 to $\frac{1}{3} \mathrm{ML}$.

The (311) surface of GaAs was reported to show a spontaneous corrugation, ${ }^{14}$ which would lead to the formation of quantum wires during thin-film growth. However, our RDS data show that the observed anisotropy is within the intrinsic anisotropy of (311)-oriented QW, indicating that the InAs layer form nearly isotropic islands with little lateral patterning due to the (possible) interface corrugation. This result is consistent with the observations of reflection high-energy electron diffraction. ${ }^{15}$ In contrast, InAs wirelike islands was observed when submonolayer InAs was grown on (100) GaAs surface. ${ }^{16}$ The observed strong RDS resonances (about 0.02 for $\Delta \rho$, which is about 60 times larger than our result) from a 0.3-ML InAs in (100) GaAs (Ref. 3) also confirmed the wirelike island formation. The growth mode of InAs submonolayer in Ref. 3 is therefore very different from ours. This is probably due to the differences in growth condition between our work and theirs. Further study is on the way to understand the influence of growth conditions on the shape of the InAs islands.

One question of which the answer is still under debate is whether ultrathin InAs layers behave like two-dimensional QW's or isolated clusters. Alonso, Ilg, and Ploog ${ }^{1}$ showed that for coverage above $\frac{1}{3}$ ML the InAs layers behaved like QW's while below 1/3 ML they were better described by isolated clusters, and there was no significant difference between the (100) and the (311) orientation. On the other hand, Yuan et al. ${ }^{5}$ showed that even at $\frac{1}{12}$ ML the (100) InAs layer was still QW-like. We propose that the discrepancy is caused by the size of the InAs islands which depends on the details of growth processes and conditions. When the size of the islands is small, the average distance between islands is also small while the electronic wave function in each island is less confined, causing a significant overlap of the wave function between adjacent islands, and the layer is QW like. On the other hand, if the individual size of the InAs islands is 
large, the wave function of each island is more confined within the island while the average distance between islands is large. The wave function overlap is much reduced and the system behaves like isolated clusters. We believe that the average size of the InAs islands in our samples are smaller than the ones in Refs. 3 and 6, because the HH peak in our 1-ML sample is about $30 \mathrm{meV}$ higher than the one reported in Ref. 6, and about $25 \mathrm{meV}$ higher than the 0.9-ML sample in Ref. 3. This could be the reason why our 1/3 ML sample is still QW like.

In summary, we have studied the optical anisotropy of submonolayer InAs inserted in the (311) GaAs matrix. Two anisotropic resonances associated with the $\mathrm{HH}$ and the $\mathrm{LH}$ of the InAs layers are observed. The steplike DOS directly demonstrates that the submonolayer InAs inserted in (311) GaAs behaves like a two-dimensional QW even at 1/3 ML coverage. The degree of anisotropy obtained from our experimental data is within the expected intrinsic anisotropy of (311)-oriented QW's, indicating that there is little structural or strain anisotropy of the ultrathin InAs inserted in the (311) GaAs matrix. Finally, we propose that whether submonolayer InAs is QW-like or clusterlike depends on the size of the InAs islands.

This work was supported by the Research Grant Council Grant No. 609/95P from the Hong Kong Government, and the experiments were performed in the William Mong's Semiconductor Cluster Laboratory at Hong Kong University of Science and Technology.

${ }^{7}$ I. Kamiya, D. E. Aspnes, L. T. Florez, and J. P. Harbinson, Phys. Rev. B 46, 15894 (1992).

${ }^{8}$ S. E. Acosta-Ortiz and A. Lanstras-Martinez, Phys. Rev. B 40, 1426 (1989).

${ }^{9}$ Z. Yang, I. K. Sou, Y. H. Yeoug, G. K. L. Wong, J. Wang, C. C. Jin, and X. Y. Hou, J. Vac. Sci. Technol. B 14, 2973 (1996).

${ }^{10}$ Tak-Kun Kwok and Z. Yang, J. Appl. Phys. 80, 4621 (1996).

${ }^{11}$ Y. Kajikawa, O. Brandt, K. Kanamoto, and N. Tsukada, J. Cryst. Growth 150, 431 (1995).

${ }^{12}$ R. Winkler and A. I. Nesvizhskii, Phys. Rev. B 53, 9984 (1996).

${ }^{13}$ W. Langbein, D. Lüer $\beta$ en, H. Kalt, J. M. Hvam, W. Braun, and K. Ploog, Phys. Rev. B 54, 10784 (1996).

${ }^{14}$ R. Nötzel, L. Däweritz, and K. Ploog, Phys. Rev. B 46, 4736 (1992).

${ }^{15}$ M. Ilg, R. Nötzel, and K. Ploog, Appl. Phys. Lett. 62, 1472 (1993).

${ }^{16}$ V. Bressler-Hill, A. Lorke, S. Varma, P. M. Petroff, K. Pond, and W. H. Weinberg, Phys. Rev. B 50, 8479 (1994). 